Article

\title{
Exploiting the Nonlinear Dynamics of Optically Injected Semiconductor Lasers for Optical Sensing
}

\author{
Maria S. Torre ${ }^{1}(\mathbb{D}$ and Cristina Masoller $2, *$ (i) \\ 1 Instituto de Física Arroyo Seco and CIFICEN (UNCPBA-CICPBA-CONICET), Universidad Nacional del \\ Centro de la Provincia de Buenos Aires, Tandil 7000, Argentina; marita@exa.unicen.edu.ar \\ 2 Departament de Física, Universitat Politècnica de Catalunya, Rambla St. Nebridi 22, \\ 08222 Terrassa, Barcelona, Spain \\ * Correspondence: cristina.masoller@upc.edu
}

Received: 26 March 2019; Accepted: 19 April 2019; Published: 24 April 2019

\begin{abstract}
Optically injected semiconductor lasers are known to display a rich variety of dynamic behaviours, including the emission of excitable pulses, and of rare giant pulses (often referred to as optical rogue waves). Here, we use a well-known rate equation model to explore the combined effect of excitability and extreme pulse emission, for the detection of variations in the strength of the injected field. We find parameter regions where the laser always responds to a perturbation by emitting an optical pulse whose amplitude is above a pre-defined detection threshold. We characterize the sensing capability of the laser in terms of the amplitude and the duration of the perturbation.
\end{abstract}

Keywords: semiconductor lasers; nonlinear dynamics; optical injection

\section{Introduction}

Complex dynamical systems often exhibit extreme or rare events. Examples in nature include earthquakes, hurricanes, financial crises, and epileptic attacks, to name just a few [1]. In recent years the generation of extreme events in optical systems has attracted attention [2,3], as such systems serve as experimental platforms for testing the physics of extreme event generation in a controlled environment, where parameters can be tuned with high precision. In particular, the dynamics of continuous-wave (cw) optically injected semiconductor lasers has attracted attention, because, under appropriated conditions, the laser can emit excitable pulses [4,5], or rare giant pulses [6]. Thus, the cw optically injected laser has been used for testing methods either to suppress [7] or to generate "on demand" [8] high optical pulses. In addition, in contrast to what can be achieved in other fields, optics laser systems allow to record long datasets containing large numbers of extreme events. Such optical "big data" has also been used for testing data analysis tools for extreme event prediction [9-11].

Here, we study the optical pulses emitted by a cw optically injected laser with a different motivation: we aim at exploiting the capability of high-pulse emission for implementing a laser-based sensor, able to detect perturbations of the strength of the injected optical field. Let us assume, for example, an optical perturbation due to the presence, during a certain time interval, of gas molecules in the beam path from the pump laser (master) to the injected laser (slave) which, due to light absorption, decrease the injected power. We aim to find appropriated conditions such that the decrease of the injected power triggers the emission of an optical pulse, high enough to cross a pre-defined "detection threshold". In order to precisely detect the optical perturbation, the emission of the pulse should occur shortly after the injected power decreases, i.e., within a pre-defined "detection time interval". In this way, the high pulse emitted will allow detecting the presence of gas molecules in the master-slave beam path. In other words, our goal is to exploit the laser excitable response for the detection of a variation of a control parameter, specifically, the decrease of the injected power. In order 
to avoid the detection of "false positives" we consider parameters such that the laser intensity, under constant injection conditions, is either constant or displays small oscillations, below the detection threshold. We show that for appropriated parameters, the decrease of the injected power can be reliably detected as it will trigger, with probability equal or close to one, the emission of a pulse, high enough to cross the detection threshold, and emitted shortly after the perturbation begins (i.e., within the detection time interval).

This paper is organized as follows. Section 2 presents the model equations, Section 3 presents the numerical results and Section 4 presents the discussion.

\section{Model}

The equations describing the dynamics of an optically injected semiconductor laser are [12-14]:

$$
\begin{aligned}
\frac{d E}{d t} & =\kappa(1+i \alpha)(N-1) E+i \Delta \omega E+\sqrt{P_{i n j}} \\
\frac{d N}{d t} & =\gamma_{N}\left(\mu-N-N|E|^{2}\right) .
\end{aligned}
$$

Here $E$ is the slow envelope of the complex optical field, $S=|E|^{2}$ is the intensity, $N$ is the carrier density, $\kappa$ is the field decay rate, $\alpha$ is the line-width enhancement factor, and $\gamma_{N}$ is the carrier decay rate. $\Delta v=\Delta \omega / 2 \pi$ with $\Delta \omega=\omega_{s}-\omega_{m}$ is the frequency detuning between the slave laser and the master laser, $P_{i n j}$ is the injection strength and $\mu$ is the injection current parameter (normalized such that the threshold of the free-running laser is at $\mu_{t h}=1$ ).

We consider a decrease of $P_{i n j}$ at time $T_{p}$ that has a Gaussian temporal shape centered at $T_{p}$, amplitude, $\Delta P$, and duration, $\Delta T: P_{i n j}(t)=P_{0}-\Delta P \exp \left[-\left(t-T_{p}\right)^{2} /\left(2 \Delta T^{2}\right)\right]$. To avoid numerical problems we take $P_{i n j}=P_{0}$ constant for $t>>T_{p}$ and $t<<T_{p}$.

We note that spontaneous emission noise is not included in the model. This is because noise can trigger the emission of pulses, which will lead to false detections. Further testing using realistic noise levels is of course necessary, in order to find model parameters such that the laser-based sensor is robust to noise. This requires that the laser dynamics have reduced sensitivity to random fluctuations [15], while it has enhanced sensitivity to deterministic perturbations of the injected optical field [16].

\section{Results}

The model was simulated with a 4th order Runge-Kutta method with an integration step of 1 ps and the parameters indicated in Table 1. In order to find appropriated injection parameters, first we varied $\Delta \omega$ and $P_{i n j}=P_{0}$ (no perturbation was applied, i.e., $\Delta P=0$ ), and for each set of parameters long time traces of the intensity dynamics were simulated $(5 \mu \mathrm{s})$, and the maximum, $I_{\max }$, and the average, $\langle I\rangle$, intensity value were calculated.

Table 1. Parameters used in the model simulations [6].

\begin{tabular}{ccc}
\hline Name & Symbol & Value \\
\hline Field decay rate & $\kappa$ & $300 \mathrm{~ns}^{-1}$ \\
Line-width enhancement factor & $\alpha$ & 3 \\
Carrier decay rate & $\gamma_{N}$ & $1 \mathrm{~ns}^{-1}$ \\
Injection current parameter & $\mu$ & 1.96 \\
Frequency detuning & $\Delta v$ & variable \\
Unperturbed injected Power & $P_{0}$ & variable \\
Perturbation amplitude & $\Delta P$ & variable \\
Perturbation duration & $\Delta T$ & variable \\
Detection time interval & $\Delta T_{\text {det }}$ & variable \\
\hline
\end{tabular}

The results are presented in Figure 1 which displays, in color code, the relative height of the intensity oscillations, $\left.\Delta I=I_{\max }-\left\langle I_{\max }\right\rangle\right) /\left\langle I_{\max }\right\rangle$ (where $I_{\max }$ is the height of the highest peak found, 
and $\left\langle I_{\max }\right\rangle$ is the average peak height) as a function of $\Delta v$ and $P_{i n j}$. The dark regions indicate either injection locking (in the region starting at $\Delta v=0$, right of the red-orange central region, the intensity is constant and there are no oscillations, $I_{\max }=\left\langle I_{\max }\right\rangle=0$ ) or period-one solutions (in the dark region to the left of the red-orange region the intensity dynamics consists of regular oscillations, all the intensity peaks are equal, $I_{\max }=\left\langle I_{\max }\right\rangle$ and $\left.\Delta I=0\right)$.

To operate the laser as a sensor, we need to select an appropriated detection threshold, $\mathrm{TH}$, such that when the injected optical power is constant, the laser intensity is either constant, or displays oscillations which are always below the detection threshold. In this way, we avoid the detection of "false positives": if $P_{i n j}$ is constant, $I(t)<T H \forall t$. In Figure 1 we see that $\Delta I<4$. Therefore, we chose a detection threshold proportional to the mean value of the height of the peaks, $T H=(1+c)\left\langle I_{\text {max }}\right\rangle$, with $c \leq 4$ being a constant that depends on the parameters. We exclude parameters for which the distribution of intensity values is long-tailed (i.e., where the laser emits rare giant pulses [6]), because for such parameters, a very high threshold will be needed in order to avoid false detections; however, a very high threshold might not detect some of the pulses that can be emitted in response to variations of $P_{i n j}$. In the following we consider the following parameters: $P_{0}=50 \mathrm{~ns}^{-2}, \Delta v=-2.29 \mathrm{GHz}$ (indicated with a circle in Figure 1) which are close to the boundary of the region where large pulses are emitted, and arbitrarily fix the threshold to $T H=2\left\langle I_{\max }\right\rangle$ (while a systematic study is needed to determine the optimal choice, our simulations suggest that the results are robust with respect to small variations of the threshold).

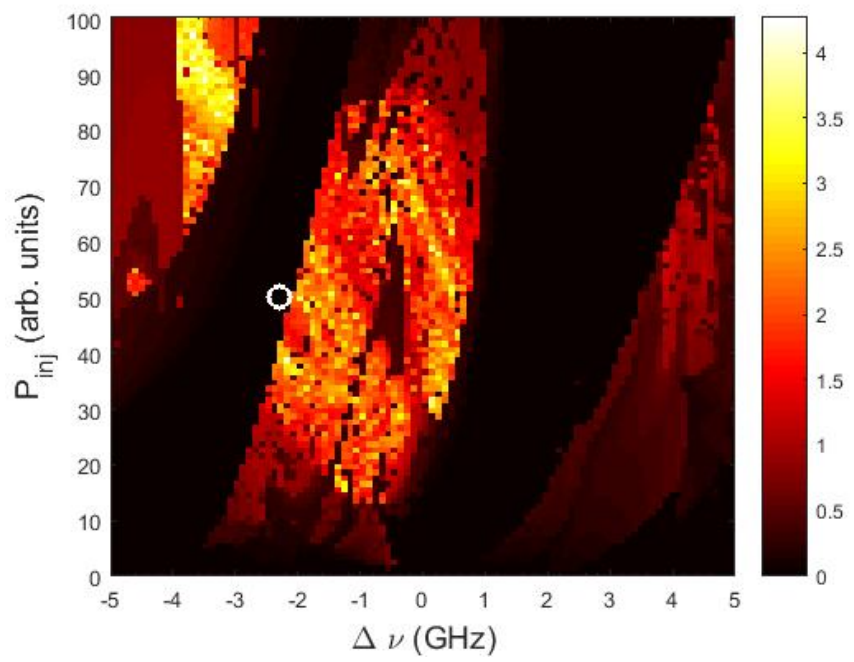

Figure 1. Relative height of the intensity oscillations when no perturbation is applied $(\Delta P=0)$, as a function of the frequency detuning, $\Delta v$, and the injection strength, $P_{i n j}$. The color code displays $\Delta I=\left(I_{\max }-\left\langle I_{\max }\right\rangle\right) /\left\langle I_{\max }\right\rangle$, with $I_{\max }$ and $\left\langle I_{\max }\right\rangle$ being the maximum and the average height of the intensity oscillations, respectively; the symbol indicates the parameters used in Figure 2: $P_{i n j}=P_{0}=$ $50 \mathrm{~ns}^{-2}$ and $\Delta v=-2.29 \mathrm{GHz}$.

Figure 2 displays two examples of the intensity time series together with the perturbation of the injected power. If, within a given detection time interval, $\Delta T_{\text {det }}$, the emitted pulses are below the threshold $T H$, the perturbation is not detected (panel a), but if at least one pulse is above $T H$, the detection is successful (panel b). As it will be discussed latter, $\Delta T_{\text {det }}$ is an important parameter of the detection system. It starts when $P_{i n j}$ decreases below a given percentage of $P_{0}$, here taken as $20 \%$. 

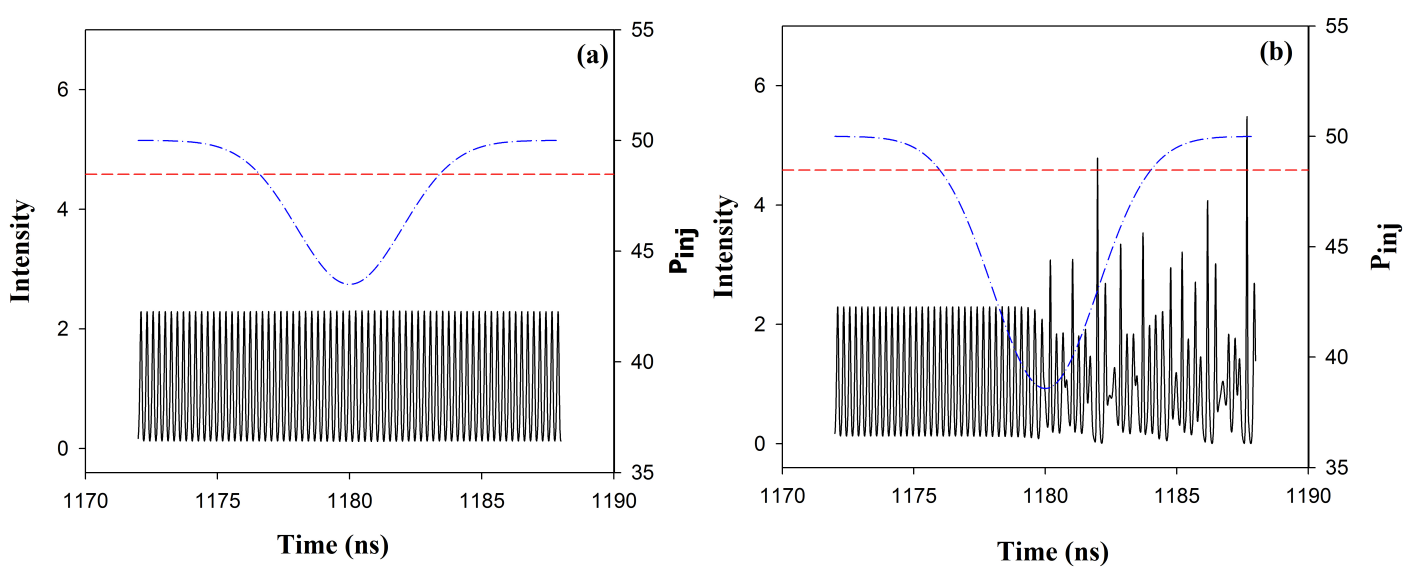

Figure 2. Time series of the laser intensity when the variation of the injected power is $\Delta P=6.6 \mathrm{~ns}^{-2}$ (a) and $\Delta P=11.4 \mathrm{~ns}^{-2}(\mathbf{b})$. In panel (a) we see that the variation is small and the intensity is always below the threshold, therefore, the variation of $P_{i n j}$ is not detected. In contrast, in panel (b), $\Delta P$ is large enough to trigger the emission of intensity pulses that are high enough to cross the threshold (indicated with a dashed line).

In order to characterize the sensing capability of the laser, we analyze the effect of the perturbation parameters: the amplitude, $\Delta P$, and the duration, $\Delta T$. Figure 3 displays the success rate, $S R$, which is the percentage of successful detections, as a function of $\Delta P$ and $\Delta T$. In this plot, the $S R$ is computed from 50 time-series with random initial conditions, and we have verified that a larger number of simulations give very similar results. We note that if the duration of the perturbation is too short, in general the detection fails because the laser has no time to respond to the perturbation by emitting a pulse that is high enough. In the other limit, if the duration of the perturbation is too long, the detection also fails, now due to the fact that the detection time interval, $\Delta T_{\text {det }}$ is too short and the laser emits a pulse at a later time. In between these two limits (if the duration of the perturbation, $\Delta T$, is not too slow nor too long with respect to the laser response time and to the detection time interval), we see in Figure 3 that the success rate is close to 1 . By increasing $\Delta T_{\text {det }}$ we improve the detection of slow perturbations, however, the minimum perturbation amplitude that is detected remains nearly unchanged. This is a consequence of the excitable nature of the dynamics: the perturbation has to be strong enough to trigger a response.
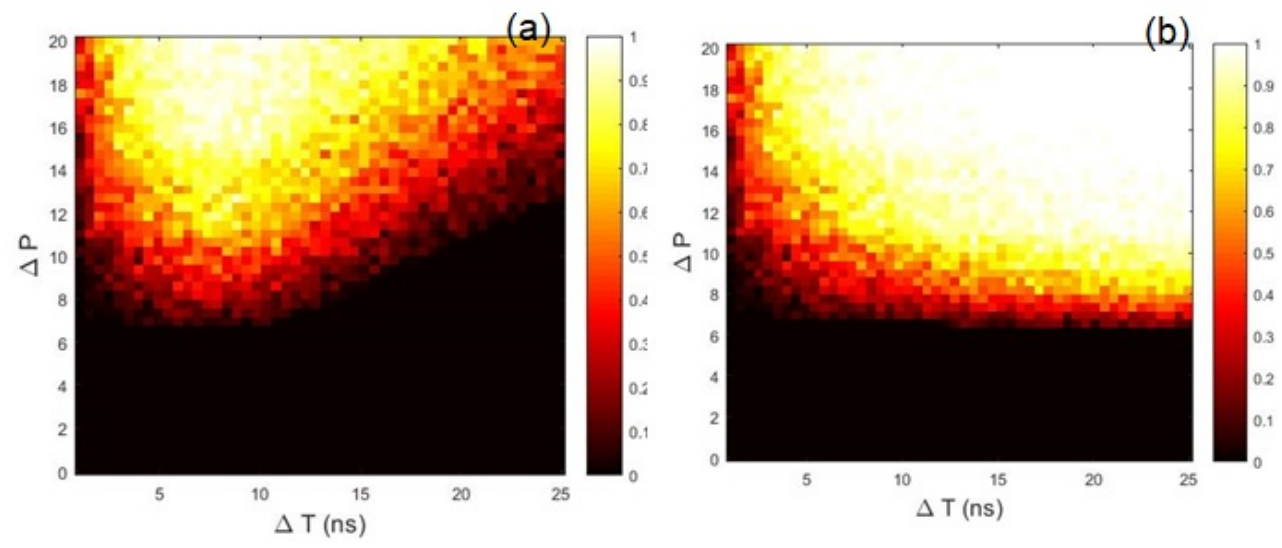

Figure 3. Success rate as a function of the perturbation amplitude, $\Delta P$, and duration, $\Delta T$. The detection time interval is $\Delta T_{\text {det }}=20 \mathrm{~ns}(\mathbf{a}), 100 \mathrm{~ns}(\mathbf{b})$. Other model parameters are $P_{i n j}=50 \mathrm{~ns}^{-2}, \mu=1.75$, and $\Delta v=-1.31 \mathrm{GHz}$. 
As shown in Figures 4 and 5 the boundary between $S R=0$ and $S R=1$ can be very sharp: if the perturbation $\Delta P$ is small and $P_{i n j}$ remains above a certain value (here $P_{i n j}>P_{i n j}^{*}=43 \mathrm{~ns}{ }^{-2}$ ) the intensity dynamics remains unaffected. On the contrary, if the perturbation is such that $P_{i n j}$ decreases below $P_{i n j}^{*}$, then pulses are emitted, which can be detected by selecting appropriated values of the threshold and of the detection time interval.
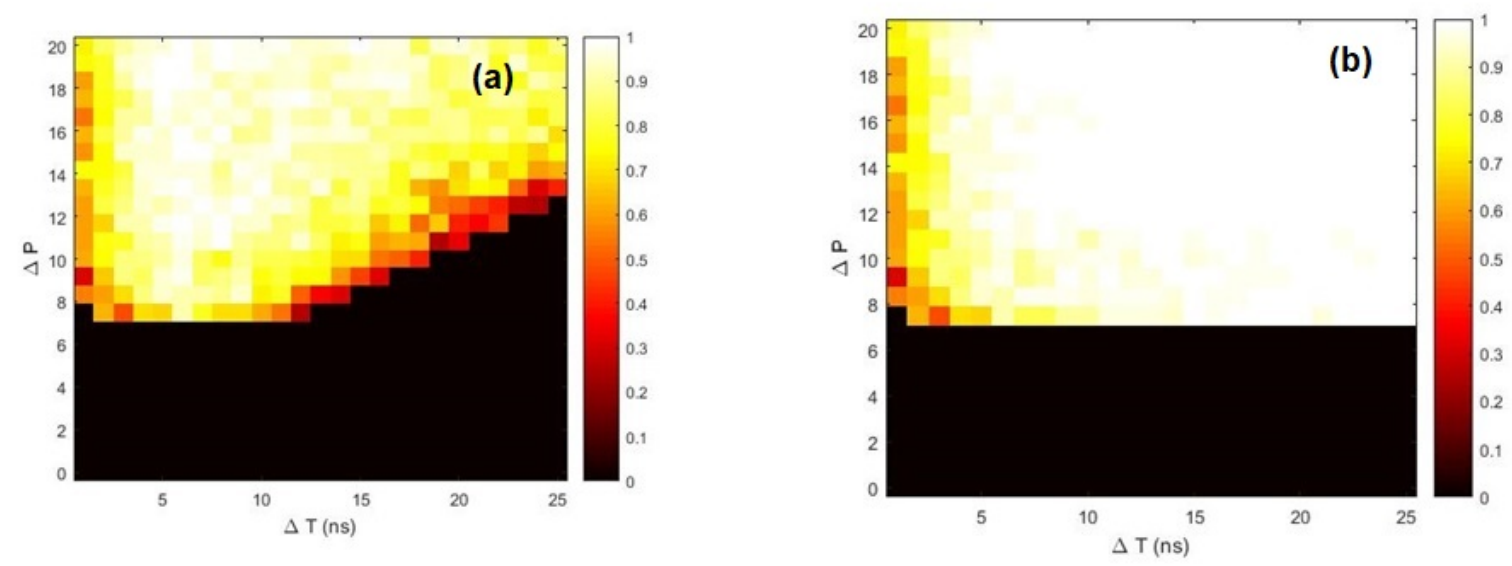

Figure 4. Success rate when the detection time interval is $\Delta T_{\text {det }}=20 \mathrm{~ns}$ (a) and $100 \mathrm{~ns}$ (b). The parameters are $P_{i n j}=50 \mathrm{~ns}^{-2}, \mu=2.064$ and $\Delta v=-2.589 \mathrm{GHz}$.
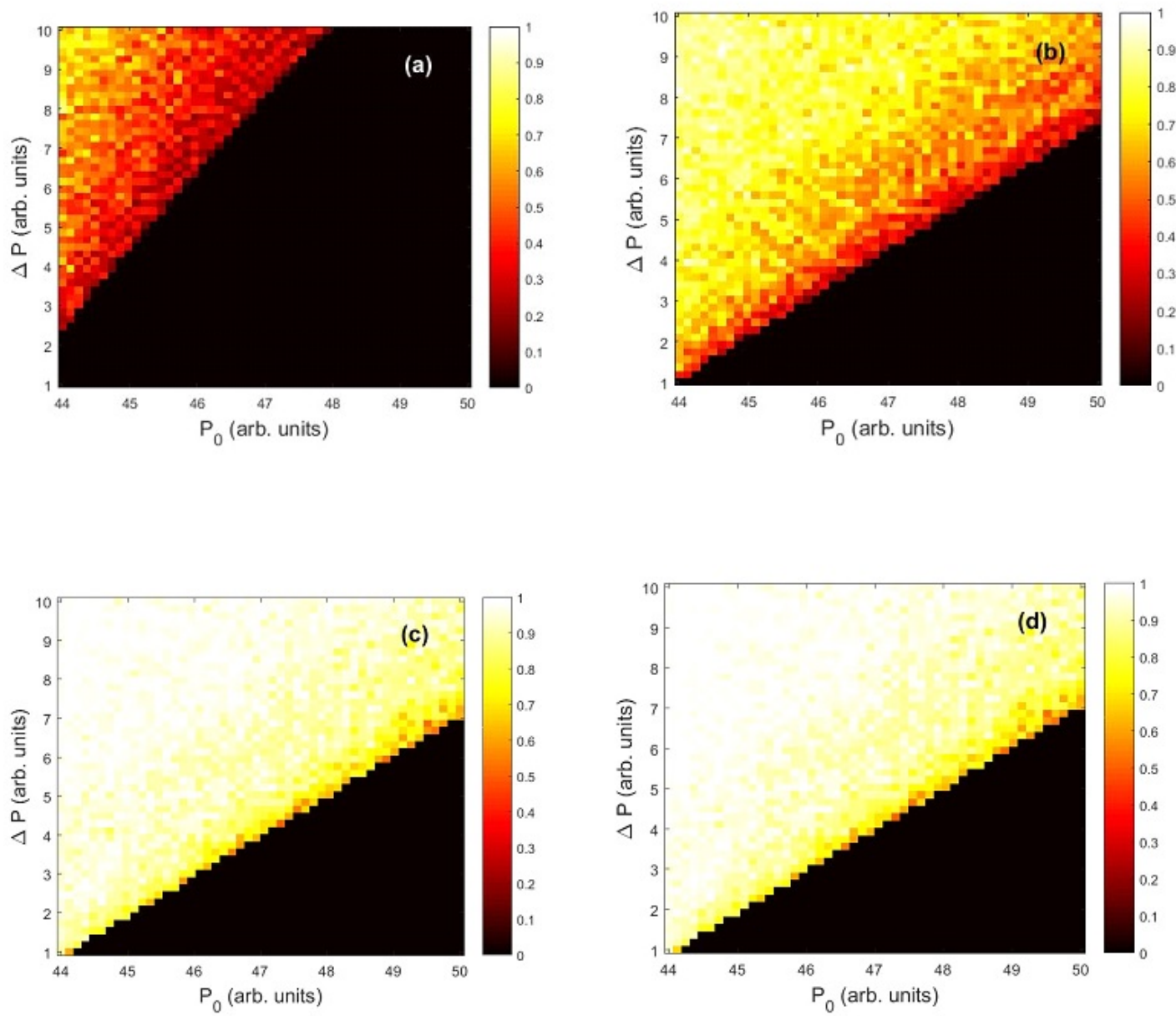

Figure 5. Success rate as a function of $P_{0}$ and $\Delta P$. The duration of the perturbation is $\Delta T=5 \mathrm{~ns}(\mathbf{a})$, $10 \mathrm{~ns}(\mathbf{b}), 20 \mathrm{~ns}(\mathbf{c}), 30 \mathrm{~ns}(\mathbf{d})$. The detection time interval is $5 \mathrm{~ns}$. 


\section{Discussion}

We have numerically studied the dynamics of an optically injected laser and have shown that, under appropriated conditions, a decrease of the injected power can be detected by the emission of optical pulses that are high enough to cross a pre-defined detection threshold, and that are emitted within a pre-defined detection time interval. The model parameters need to be chosen such that the laser intensity, under constant injected power, has a well-defined maximum value (i.e., the distribution of intensity values does not exhibit a long tail). In this case, a detection threshold can be defined such that, in the absence of perturbation, the intensity oscillations are always below the threshold, while at least one intensity pulse crosses the threshold with probability close or equal to one, if a perturbation is applied such that the injected power decreases. We have studied the limitations regarding the amplitude and the duration of the perturbation. In general, due to the excitable nature of the dynamics, the amplitude of the perturbation needs to be large enough, while its duration needs to be not too short nor too long. If the perturbation is too fast, the laser has no time to respond by emitting a pulse high enough, while if the perturbation is too long, the emitted pulse can be delayed with respect to the detection time interval.

In this study we have considered a Gaussian shape for the perturbation, and it will be important, for practical applications, to test the performance of the sensor using different shapes and to analyze how the detection threshold and the detection time depend on the shape of the perturbation. We have simulated noise-free equations to avoid detecting noise-induced pulses as "false positives". Further testing using realistic noise levels is of course necessary, in order to find model parameters such that the laser dynamics is robust to noise, while is sensitive to deterministic perturbations of the injected field. An interesting setup to analyze is that of ultra-short optical feedback [17]. Further work will probably also aim to compare the detection method proposed here, which exploits the excitable properties of the laser dynamics, with more traditional approaches for sensing.

Author Contributions: M.S.T. and C.M. designed the study; M.S.T. performed the simulations; both authors discussed the results and wrote the manuscript.

Funding: C.M. was funded in part by a SANTANDER INVESTIGACION grant and a Spanish MINECO/FEDER grant (FIS2015-66503-C3-2-P).

Conflicts of Interest: The authors declare no conflict of interest. The funders had no role in the design of the study; in the collection, analyses, or interpretation of data; in the writing of the manuscript, or in the decision to publish the results.

\section{References}

1. Albeverio, S.; Jentsch, V.; Kantz, H. Extreme Events in Nature and Society; Springer: Berlin/Heidelberg, Germany, 2006; ISBN 978-3-540-28611-0.

2. Solli, D.R.; Ropers, C.; Koonath, P.; Jalali, B. Optical rogue waves. Nature 2007, 450, 1054-1057. [CrossRef] [PubMed]

3. Akhmediev, N.; Kibler, B.; Baronio, F.; Belić, M.; Zhong, W.P.; Zhang, Y.; Chang, W.; Soto-Crespo, J.M.; Vouzas, P.; Grelu, P.; et al. Roadmap on optical rogue waves and extreme events. J. Opt. 2016, 18, 063001. [CrossRef]

4. Goulding, D.; Hegarty, S.P.; Rasskazov, O.; Melnik, S.; Hartnett, M.; Greene, G.; McInerney, J.G.; Rachinskii, D.; Huyet, G. Excitability in a Quantum Dot Semiconductor Laser with Optical Injection. Phys. Rev. Lett. 2007, 98, 153903. [CrossRef] [PubMed]

5. Turconi, M.; Garbin, B.; Feyereisen, M.; Giudici, M.; Barland, S. Control of excitable pulses in an injection-locked semiconductor laser. Phys. Rev. E 2013, 88, 022923. [CrossRef] [PubMed]

6. Bonatto, C.; Feyereisen, M.; Barland, S.; Giudici, M.; Masoller, C.; Leite, J.R.; Tredicce, J.R. Deterministic Optical Rogue Waves. Phys. Rev. Lett. 2011, 107, 053901. [CrossRef] [PubMed]

7. Zamora-Munt, J.; Garbin, B.; Barland, S.; Giudici, M.; Leite, J.R.; Masoller, C.; Tredicce, J.R. Rogue waves in optically injected lasers: Origin, predictability, and suppression. Phys. Rev. A 2013, 87, 035802. [CrossRef] 
8. Jin, T.; Siyu, C.; Masoller, C. Generation of extreme pulses on demand in semiconductor lasers with optical injection. Opt. Express 2017, 25, 031326. [CrossRef] [PubMed]

9. Birkholz, S.; Brée, C.; Demircan, A.; Steinmeyer, G. Predictability of Rogue Events. Phys. Rev. Lett. 2015, 114, 213901. [CrossRef] [PubMed]

10. Martinez Alvarez, N.; Borkar, S.; Masoller, C. Predictability of extreme intensity pulses in optically injected semiconductor lasers. Eur. Phys. J. Spec. Top. 2017, 226, 1971-1977. [CrossRef]

11. Colet, M.; Aragoneses, A. Forecasting Events in the Complex Dynamics of a Semiconductor Laser with Optical Feedback. Sci. Rep. 2018, 8, 10741. [CrossRef] [PubMed]

12. Wieczorek, S.; Krauskopf, B.; Simpson, T.B.; Lenstra, D. The dynamical complexity of optically injected semiconductor lasers. Phys. Rep. 2005, 416,1-128. [CrossRef]

13. Kovanis, V.; Gavrielides, A.; Gallas, J.A.C. Labyrinth bifurcations in optically injected diode lasers. Eur. Phys. J. D 2010, 58, 181-186. [CrossRef]

14. Ohtsubo, J. Semiconductor Lasers: Stability, Instability and Chaos; Springer: Berlin/Heidelberg, Germany, 2012; ISBN 978-3-642-30147-6.

15. Simpson, T.B.; Liu, J.M.; AlMulla, M.; Usechak, N.G.; Kovanis, V. Limit-Cycle Dynamics with Reduced Sensitivity to Perturbations. Phys. Rev. Lett. 2014, 112, 023901. [CrossRef] [PubMed]

16. Torre, M.S.; Masoller, C.; Mandel, P.; Shore, K.A. Enhanced sensitivity to current modulation near dynamic instability in semiconductor lasers with optical feedback and optical injection. J. Opt. Soc. Am. B 2004, 21, 302-306. [CrossRef]

17. Lo, K.H.; Hwang, S.K.; Donati, S. Numerical study of ultrashort-optical-feedback-enhanced photonic microwave generation using optically injected semiconductor lasers at period-one nonlinear dynamics. Opt. Express 2017, 25, 31595-31611. [CrossRef] [PubMed]

(C) 2019 by the authors. Licensee MDPI, Basel, Switzerland. This article is an open access article distributed under the terms and conditions of the Creative Commons Attribution (CC BY) license (http:/ / creativecommons.org/licenses/by/4.0/). 